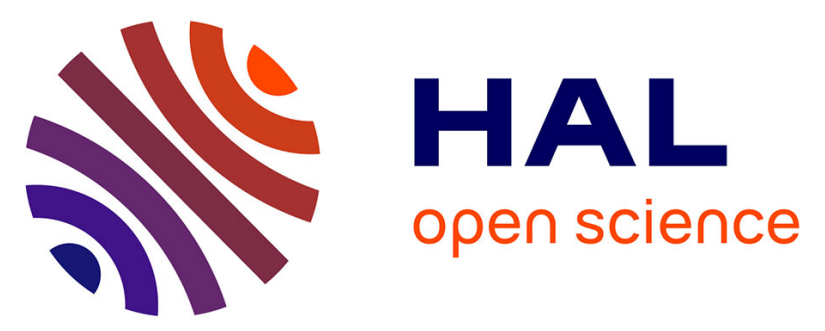

\title{
A first wide-open LDH structure hosting InP/ZnS QDs: \\ a new route toward efficient and photostable red-emitting phosphor
}

Rodolphe Valleix, Qian Zhang, Damien Boyer, Philippe Boutinaud, Geneviève Chadeyron, Yongjun Feng, Hanako Okuno, François Réveret, Horst

Hintze-bruening, Fabrice Leroux

\section{To cite this version:}

Rodolphe Valleix, Qian Zhang, Damien Boyer, Philippe Boutinaud, Geneviève Chadeyron, et al.. A first wide-open LDH structure hosting InP/ZnS QDs: a new route toward efficient and photostable red-emitting phosphor. Advanced Materials, 2021, 33, pp.2103411. 10.1002/adma.202103411 . hal03318403

\section{HAL Id: hal-03318403 \\ https://hal.uca.fr/hal-03318403}

Submitted on 10 Sep 2021

HAL is a multi-disciplinary open access archive for the deposit and dissemination of scientific research documents, whether they are published or not. The documents may come from teaching and research institutions in France or abroad, or from public or private research centers.
L'archive ouverte pluridisciplinaire $\mathbf{H A L}$, est destinée au dépôt et à la diffusion de documents scientifiques de niveau recherche, publiés ou non, émanant des établissements d'enseignement et de recherche français ou étrangers, des laboratoires publics ou privés. 


\section{A First Wide-Open LDH Structure Hosting InP/ZnS QDs: A New Route Toward Efficient and Photo-Stable Red-Emitting Phosphor}

Rodolphe Valleix, Qian Zhang, Damien Boyer,* Philippe Boutinaud, Geneviève Chadeyron, Yongjun Feng, Hanako Okuno, François Réveret, Horst Hintze-Bruening, Fabrice Leroux*

R. Valleix, Q. Zhang, Dr. D. Boyer, Prof. G. Chadeyron, Prof. P. Boutinaud, Dr. F. Leroux, Université Clermont Auvergne, Clermont Auvergne INP, CNRS, ICCF, F-63000 Clermont-Ferrand, France.

E-mail: fabrice.leroux@uca.fr, damien.boyer@sigma-clermont.fr

Q. Zhang, Dr. Y. Feng, State Key Laboratory of Chemical Resource Engineering, Beijing Engineering Center for Hierarchical Catalysts, Beijing University of Chemical Technology, No. 15 Beisanhuan East Road, Beijing 100029, China.

Dr. H. Okuno, Université Grenoble Alpes, CEA, IRIG-MEM, 38000 Grenoble, France.

Dr. F. Réveret, Université Clermont Auvergne, Clermont Auvergne INP, CNRS, Institut Pascal, F63000 Clermont-Ferrand, France.

Dr. H. Hintze-Bruening, Paderborn University, Y-0.211, Mersinweg 7, 33098 Paderborn, Germany.

Keywords: Indium phosphide, Quantum dots, Bola-amphiphiles, Layered double hydroxide, Silicone composite film, Red-emitting phosphor 


\begin{abstract}
:
The architecture of $\mathrm{Zn}-\mathrm{Al}$ layered double hydroxides (LDHs), organo-modified with bolaamphiphiles molecules, is matching its interlayer space to the size of narrow-band red-emitting InP/ZnS core-shell QDs to form original high-performance functional organic-inorganic QD-bolaLDH hybrids. The success of size-matching interlayer space (SMIS) approach is confirmed by Xray diffraction, SAXS, TEM, STEM-HAADF and photoluminescence investigations. The QD-BolaLDH hybrid exhibits a photoluminescence quantum yield three times higher than that of pristine $\mathrm{InP} / \mathrm{ZnS}$ QDs and provides an easy dispersion into silicone-based resins, what makes the SMIS approach a change of paradigm compared to intercalation chemistry using common host structures. Moreover, this novel hybrid presents low QD-QD energy transfer comparable to that obtained for QDs in suspension. Composite silicone films incorporating InP/ZnS (0.27 wt. \%) QD-bola-LDH hybrids further show remarkable improved photo-stability relative to pristine QDs. A LED overlay consisting of a blue LED chip and silicone films loaded with QD-bola-LDH hybrids and YAG:Ce phosphors exhibits a color rendering index close to 94 .
\end{abstract}


Semiconductor nanocrystals (NCs) form a well-known class of emerging materials which find applications in many forefront fields such as biological imaging/labelling, ${ }^{[1]}$ lasers, ${ }^{[2]}$ displays ${ }^{[3]}$ and solid-state lighting. ${ }^{[4]}$ Colloidal quantum dots (QDs) are among the most promising materials to integrate this technology. These NCs exhibit a three-dimensional quantum confinement which endows them with outstanding optical properties, such as size-tunable emission, in addition to presenting a broad absorption range. Environmental concerns have forced the scientific community to move from the well-established CdSe QDs to less toxic Cd-free compositions. Today, indium phosphide (InP) is one of the most-studied substitutes. Despite the fact that the synthesis of $\operatorname{InP}$ QDs was first described 25 years ago by Mićić et al., ${ }^{[5]}$ it remained scarcely used because of the pyrophoric and toxic nature of the phosphorous precursor, tris-(trimethylsilyl)-phosphine (P(TMS $\left.)_{3}\right)$. In 2013, Song et al. reported on the synthesis of InP QDs through a cost-effective and safer way using aminophosphines (e.g. $\left.\mathrm{P}\left(\mathrm{N}\left(\mathrm{CH}_{3}\right)_{2}\right)_{3}\right) .{ }^{[6]}$ This method presents an acceptable size distribution despite a photoluminescence quantum yield (PLQY) lower than that attained with $\mathrm{P}(\mathrm{TMS})_{3}$. Moreover, the main drawback of InP-based QDs is their loss of stability under continuous photonic excitation compared to Cd-based QDs. This issue can however be overcome by increasing the thickness of the shell using a core-shell structure such as InP/ZnSe. ${ }^{[7]}$ Thus a multi-shelled InP core like InP/ZnSeS/ZnS is reported to reach a near-unity PLQY with high stability under continuous UV light irradiation. ${ }^{[8]}$ So far, other structures like InP/ZnSe/ZnS ${ }^{[9]}$ and InP/GaP/ZnS ${ }^{[10]}$ exhibit good photonic stability, but their synthesis requires almost twelve hours to complete and their scale-up is highly unlikely. Another enticing approach to stabilizing InP-based QDs is to employ a protective inorganic host structure. Evidently, such a host structure must show high flexibility to accommodate the size of QDs. Hitherto, metal organic frameworks (MOFs) or layered (2D) structures have appeared as relevant host structures to embed QDs. MOFs presenting large open structures associated with high surface areas have been extensively studied, and their association with QDs, whether in an "onto" or "into" mode, find applications, as reviewed for photocatalysis, ${ }^{[11]}$ and energy ${ }^{[12]}$ as well as light-harvesting. ${ }^{[13]}$ As far as the topic of QDs armored 
by $2 \mathrm{D}$ structures is concerned, a heterogeneous hybrid structure is formed using the layer-by-layer (LbL) method. Based on electrostatic interaction, the LbL process requires monolayers or highlydispersed sheets to fabricate films to be implemented into optical devices. In combination with a 2D structure and exemplified by layered double hydroxide (LDH), the LbL approach involves positively-charged self-assembled rigid platelets alternating with soft organic segments, usually a polymer, where QDs are encapsulated, as exemplified by $\mathrm{CdSe} / \mathrm{CdS} / \mathrm{ZnS}$ in poly(maleic acid-altoctadecene), ${ }^{[14]}$ CdTe and CdSe/ZnS in polyvinyl alcohol (PVA), ${ }^{[15]}$ CdTe QDs directly in the LDH interlamellar space ${ }^{[16,17]}$ or, as for dihydrolipoic acid-decorated PbS QDs and exfoliated LDH nanosheets, by drop-casting. ${ }^{[18]}$

As for the multi-shelled QD approach, LbL technology is time-consuming, and probably limited to the fabrication of thin films; moreover, the exfoliation process is also limited to small LDH concentrations in large quantities of solvent, hindering any scale-up. An alternative is to accommodate QDs directly into the LDH interlamellar space by a straightforward and scalable method. LDH-type materials are built from edge-sharing octahedral units forming positive layers by cation substitution from brucite $\mathrm{M}_{(1-\mathrm{x})}^{\mathrm{II}} \mathrm{M}^{\mathrm{III}}{ }_{\mathrm{x}}$ with the presence of the interleaved species, anions and water molecules. Usually, and as for mesoporous MOFs using large-size templating agents to entrap QDs such as perovskite QDs, ${ }^{[19]}$ surfactant molecules are selected. Such long-chain fatty acid anions, such as arachidate, are found to prop apart the layered structure. The process is of Langmuir-Blodgett (LB) ${ }^{[20]}$ type or through the delamination of dodecyl sulfate (DS) LDH exfoliated into 1-butanol and restacked onto (dodecanethiol-capped 4-nm) CdSe. ${ }^{[21]}$ Bearing in mind that QDs should be fully embedded to be protected by the mineral host, the "into" approach is preferred to immobilisation «onto » the platelets; the layered structure should then match the QDs size without being disrupted (delaminated). Usual surfactant molecules are here discarded, as they are tethered at one end-molecule only, making it possible for the sheets to delaminate and, as the size needed for the alkyl chain will be probably too long (over 5 to $6 \mathrm{~nm}$ length), thus rendering the surfactant too waxy to mix intimately with LDH platelets. As far as the size of the basal spacing 
is concerned, polymeric bola-amphiphiles, ${ }^{[22 \mathrm{a}, 22 \mathrm{~b}, 22 \mathrm{c}]}$ in particular $\alpha, \omega$-carboxylate-terminated hydrophobic backbones (Figure S1) are reported to generate an interlayer space as large as 5 to 6 $\mathrm{nm},{ }^{[23 a, 23 \mathrm{~b}]}$ close here to the size of the QDs. It is found that LDH phases are highly compatible with such bi-terminal molecules, which is reminiscent of terephthalate intercalation pioneered by Drezdon. ${ }^{[24]}$

Relying upon previous studies, ${ }^{[23 a]}$ the first QD-LDH hybrid materials are synthesized via an original method where organo-modified bola-LDH hybrid structures are placed in contact with oleylamine-capped QDs in suspension in chloroform (Figure 1). The interlayer spacing is adjusted by selecting the suitable bola-amphiphile size to load InP/ZnS QDs. The resulting composites are straightforward to produce since they are free of any QD ligand exchange procedure. This last point is in most cases a prerequisite to integrate a host structure, but it generally leads to a significant drop in the optical properties. ${ }^{[25]}$

Figure 1. Schematic representation of the synthesis of a QD-LDH composite structure. 
InP-core QDs were produced by a previously published hot-injection method by first mixing zinc and indium halides in oleylamine (OLAm). ${ }^{[26]}$ Then the phosphorous precursor was injected at high temperature, thereby producing tetrahedral zinc-blende type InP cores (ESI section 1 and Figure S2a and b). The ZnS shelling was processed by bringing the sulfur precursor to the mixture, followed by the zinc carboxylate. The temperature was then increased to $260^{\circ} \mathrm{C}$ to ensure the formation of a $\mathrm{ZnS}$ layer on the InP core (ESI section 1). Both the tetrahedral shape and the desired zinc-blende crystalline structure were preserved after shell deposition (Figure 2a, 2b and 3a).

The obtained InP/ZnS QDs were characterized by STEM-HAADF (Figure 2a and b) and powder small angle X-ray scattering (SAXS, Figure 2c) to estimate their size and size distribution. The scattering vector $\mathrm{q}_{\mathrm{QDs}}$ of the QDs obtained by SAXS has a value of $1.3 \mathrm{~nm}^{-1}$, which gives a realspace distance $\mathrm{d}_{\mathrm{QDs}}=4.9 \mathrm{~nm}$ after conversion using Equation 1 .

$$
\mathrm{d}=\frac{2 \pi}{q}
$$

This calculated dimension agrees well with the edge length of the QDs measured at $4.4 \mathrm{~nm}$ by STEM-HAADF.

Uncoated InP cores do not show any fluorescence due to the presence of under-coordinated surface bonds acting as charge carrier traps. ${ }^{[27]}$ Fluorescence appears with the ZnS shelling, which passivates dangling bonds present at the InP surface. Correspondingly, the excitonic absorption of the InP/ZnS QDs presents a half width at half maximum (HWHM $\left.{ }^{[28]}\right)$ of $34 \mathrm{~nm}(110 \mathrm{meV})$, in line with earlier values reported for these QDs, ${ }^{[29]}$ and appears to be red-shifted by $\sim 10 \mathrm{~nm}$ with respect to the InP-core excitonic transition (Figure S2c). This red shift is ascribed to exciton delocalization throughout the shell. ${ }^{[26,30]}$ The 360 nm-excited emission of well-dispersed InP/ZnS QDs suspended in chloroform at a concentration of $1.5 \mathrm{~g} . \mathrm{L}^{-1}$ consists of a single peak at $624 \mathrm{~nm}(1.99 \mathrm{eV})$, with a full width at half maximum (FWHM) of $59 \mathrm{~nm}(187 \mathrm{meV})$, see Figure 2d. This result is consistent with the literature. ${ }^{[31]}$ 
(a)

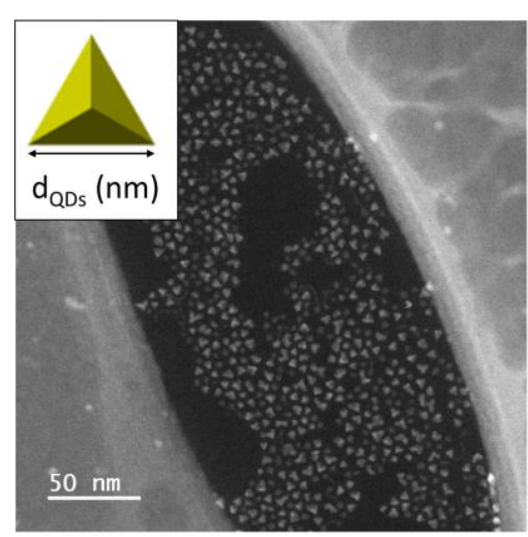

(c)

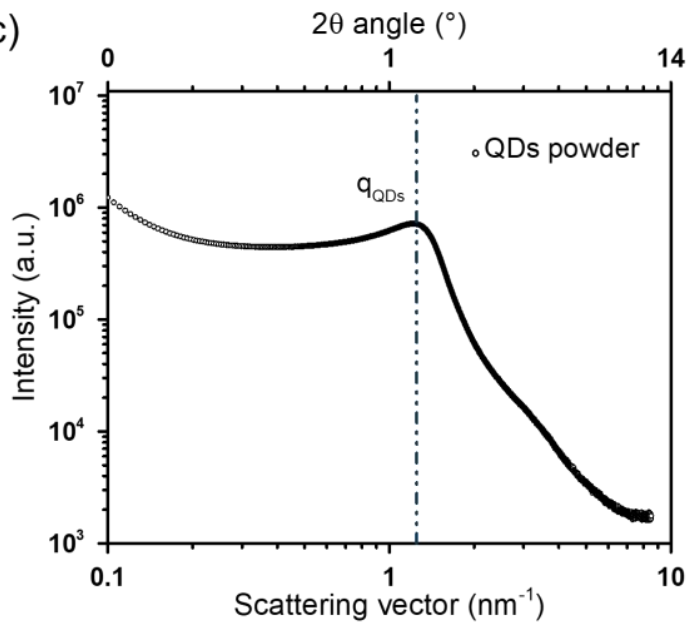

(b)

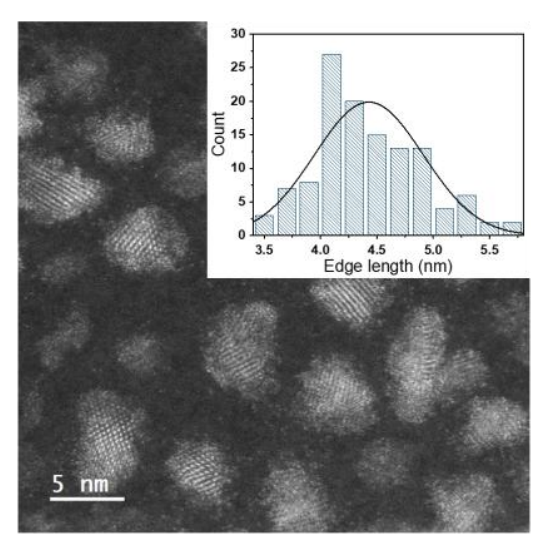

(d)

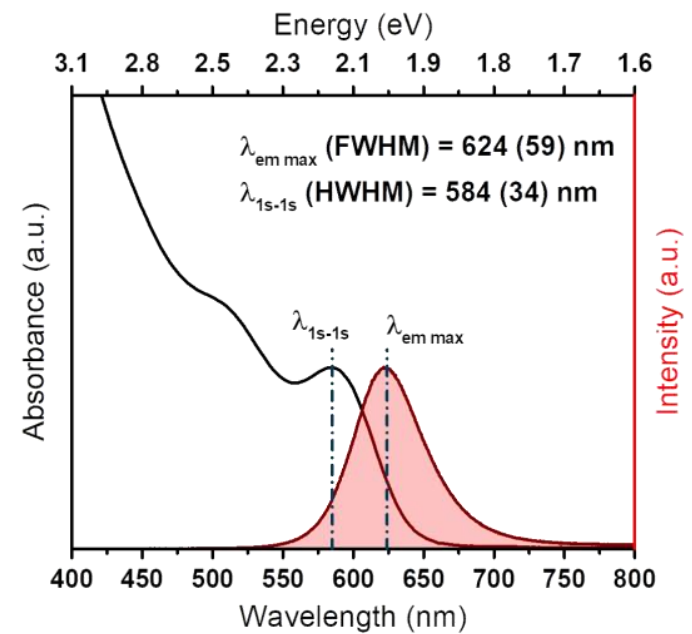

Figure 2. Representative STEM-HAADF images of the synthesized oleylamine-capped tetrahedral InP/ZnS QDs with in inset their geometrical shape (a) and a higher magnification of the sample with in inset the size distribution histogram (recorded on over 100 particles) (b), SAXS data of the same InP/ZnS QDs powder (c) absorption (black line) and emission (red line) spectra of the QDs in suspension in $\mathrm{CHCl}_{3}\left(\lambda_{\text {exc }}=360 \mathrm{~nm}\right)(\mathrm{d})$.

Bola-LDHs were synthesized using the polyol method from zinc and aluminum salts and bolaamphiphile polymer. This suspension was mixed with a suspension of InP/ZnS QDs to obtain the desired QD-bola-LDH hybrids by diffusion of the QDs into the interlamellar space.

The successful insertion of the QDs was confirmed by combining XRD and SAXS to obtain the entire sequence of the characteristic (00l) reflections of the lamellar architecture, see Figure 3. The peak positions as deduced by SAXS were collected by means of the first derivative for better precision (Figure S3). For bola-LDH, the characteristic Bragg peaks of $\mathrm{Zn}_{2} \mathrm{Al}-\mathrm{LDH}$ exhibit a series 
of basal reflections (00l) at low angles and the (110) reflection at a higher angle, indicating that a layered structure and an intralayer structural ordering are formed, respectively. The SAXS and XRD data (Table 1) indicate a layered structure with a large number of harmonic peaks resulting in a basal spacing of $6.47 \pm 0.06 \mathrm{~nm}$ and $6.92 \pm 0.02 \mathrm{~nm}$ for bola-LDH and QD-bola-LDH, respectively. These values agree with the distances measured by TEM: $6.4 \mathrm{~nm}$ for bola-LDH and $7.3 \mathrm{~nm}$ for QD-bola-LDH (Figure 3c and 3d). Interestingly, QD diffraction peaks are still observed in the hybrid structure even with a loading as low as $3 \mathrm{wt} . \%$, due to the high electronic contrast (Figure $3 \mathrm{a}$ - right). Note that the (003) reflection is hidden in the SAXS pattern (Figure 3b) because it overlaps with the scattering of larger particles (aggregates).

Table 1. Data collection reporting X-ray diffraction angles, SAXS q-values and the corresponding d-value for QD-bola-LDH and bola-LDH hybrid structures.

\begin{tabular}{c|c|c|c|c|c|c|}
\multicolumn{9}{c|}{ QD-bola-LDH } & \multicolumn{3}{c}{ bola-LDH } \\
$(\mathrm{hkl})$ & $\begin{array}{c}\text { XRD } \\
\left({ }^{\circ}\right)\end{array}$ & $\begin{array}{c}\text { SAXS } \\
\left(\mathrm{nm}^{-1}\right)\end{array}$ & $\begin{array}{c}\mathrm{d}^{\mathrm{a})} \\
(\mathrm{nm})\end{array}$ & $\begin{array}{c}\text { XRD } \\
\left({ }^{\circ}\right)\end{array}$ & $\begin{array}{c}\text { SAXS } \\
\left(\mathrm{nm}^{-1}\right)\end{array}$ & $\begin{array}{c}\left.\mathrm{d}^{\mathrm{a}}\right) \\
(\mathrm{nm})\end{array}$ \\
\hline$(003)$ & - & 0.89 & 7.06 & 1.36 & 0.98 & $6.50 / 6.41$ \\
$(006)$ & - & 1.78 & 7.06 & 2.68 & 1.94 & $6.59 / 6.48$ \\
$(009)$ & 3.81 & 2.72 & $6.96 / 6.93$ & 4.05 & 2.89 & $6.54 / 6.52$ \\
$(0012)$ & 5.08 & - & 6.96 & 5.45 & 3.87 & $6.49 / 6.49$ \\
$(0015)$ & 6.39 & - & 6.92 & 6.83 & - & 6.46 \\
$(0018)$ & 7.66 & - & 6.92 & 8.27 & - & 6.41 \\
$(0021)$ & 9.01 & - & 6.87 & 9.64 & - & 6.42
\end{tabular}

a) Distance $d$ was calculated using Bragg equation (i.e. 2.d.sin $(\theta)=\lambda$ ) and Equation 1 from XRD data and SAXS data, respectively

For QD-bola-LDH, the Bragg reflections for both XRD and SAXS patterns are slightly broadened compared to the QD-free pristine assembly (Figure 3a and b). At first glance, this may indicate a decrease in the structural coherence length resulting from a limited number of stacked layers in the hybrid LDH. The expansion of the basal spacing from 6.47 to $6.92 \mathrm{~nm}$ is subsequent to the rearrangement of the interleaved bola anions to accommodate InP/ZnS QDs. This occurs through a 
tilting or kinking of the bi-terminal bola groups attached to the LDH lamellar network. This interlamellar length of $6.92 \mathrm{~nm}$ determined by SAXS supports the fact that QDs (and ligands after slight rearrangements) enter the interlamellar space as isolated, non-aggregated guests. The evaporating solvent method appears therefore to be a relevant route for the intercalation of QDs within the interlayer space of a highly expandable bola-LDH host. ${ }^{[32 \mathrm{a}]} \mathrm{LDH}$ is pushed here to its limit through a stable pillar-structure to fit QDs size. The innovative SMIS approach, different from classical methods using simple carbonate ${ }^{[14,32 b, 32 c]}$ or hybrid ${ }^{[21,32 d]}$ LDH phases or LbL successive depositions, ${ }^{[15,17]}$ may be considered as a breakthrough in both intercalation chemistry and polymer filler aspect for advanced materials. 
(a)
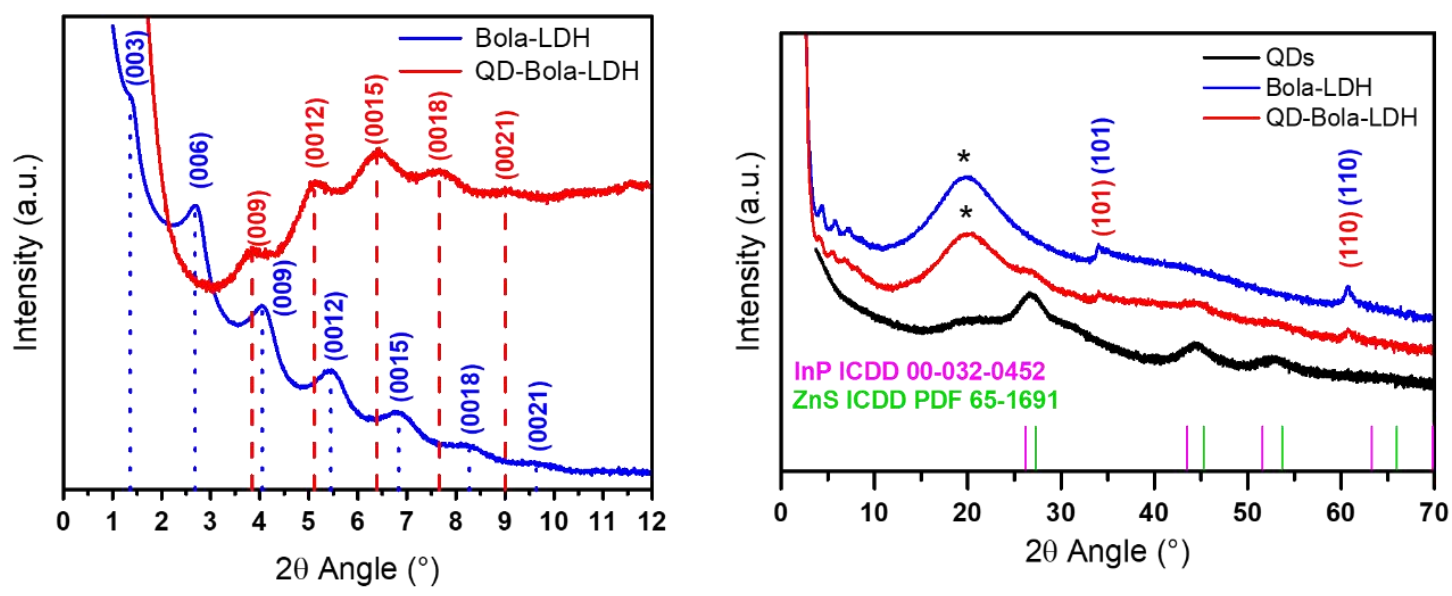

(b)

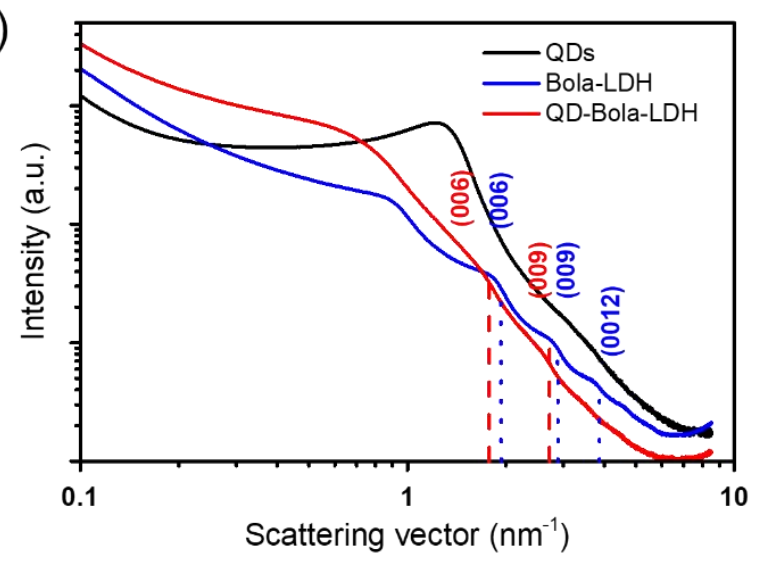

(c)

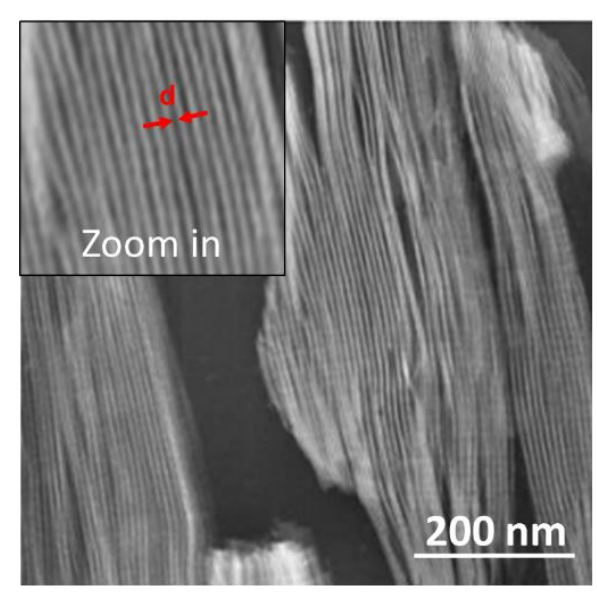

(d)

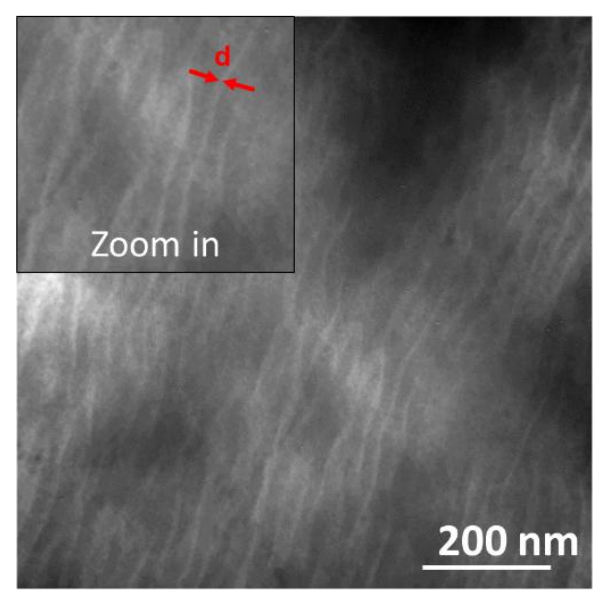

Figure 3. XRD patterns of QD-bola-LDH (red), bola-LDH (blue) powders and QD powder (black) (a - right) (the Bola contribution from its intramolecular segment-repetition is stared for clarity), XRD of bola-LDH (blue) and QD-bola-LDH (red) in the 1-12 ${ }^{\circ}$ region recorded over a period of 11 hours (a - left), SAXS data of QD-bola-LDH (red), bola-LDH (blue) powders and QD powder (b) and TEM images of the bola-LDH and QD-bola-LDH composites (c) and (d), respectively. 
Dispersing bola-LDHs in chloroform has no effect on the characteristic lamellar structure (Figure S4). Figure S5 depicts the FT-IR spectrum of bola-LDH and QD-bola-LDH compounds; no modification is noted. Since the vibrations associated with the bola backbone remain the same, this eliminates stretching as an explanation of the basal expansion and supports the fact that this expansion has a tilting origin. Considering a layer thickness and a bola length of $7.3 \mathrm{~nm},{ }^{[33]}$ the tilt angle between bola and LDH mineral network is about $39^{\circ}$. This angle drops to $26^{\circ}$ after the addition of the QDs, and confirms the success of the incorporation. Two other types of LDHs were investigated as hosts for QDs, where carbonate and dodecyl sulfate spacers were used to fabricate $\mathrm{CO}_{3}-\mathrm{LDH}$ and DS-LDH, respectively. The corresponding XRD and SAXS patterns indicate no expansion of the interlamellar distance in these cases, i.e. no incorporation of the QDs within the HDL gallery (Figure S6). The resulting powders should be seen as a physical mixture in which QDs are localized in the vicinity of the platelets in an « onto » mode rather than « into » the interlayer space. Other bi-tethered spacers, such as terephthalic acid (TA) and $\alpha, \omega$-dicarboxylic acid polyethylene glycol $\left(\mathrm{PEG}_{1000}\right)$, are employed to accommodate QDs. For TA-LDH, no changes are noticed neither on XRD nor on SAXS data after fabricating the QD-TA-LDH indicating that the QDs fail to be incorporated into the interlamellar space (Figure S7a and b). In the case of the $\mathrm{PEG}_{1000}$, home-made synthesized (ESI - section 1), presenting both the length $(8.3 \mathrm{~nm})^{[34]}$ and the $\alpha / \omega$ functionalization comparable to ones of the bola (Figure S8a), the syntheses by coprecipitation or by the polyol method fail to produce the desired hybrids. As presented on the XRD patterns (Figure S8b and c), the spacers are counter-anion of the starting salts, nitrates and acetates for co-precipitation and polyol route, respectively. However, several vibrations bands are attributed to PEG molecules on the FT-IR spectrum (Figure S8d). Indeed, PEG $_{1000}$ molecules are not rigid enough to allow them to act as spacers, their high degree of flexibility forces them instead to be adsorbed on the LDH layers, this reminiscent of superplasticizers adsorption in cement chemistry. [35] 
The photoluminescence of QD-CO $\mathrm{CO}_{3}-\mathrm{LDH}$ and QD-DS-LDH hybrid powders excited at $360 \mathrm{~nm}$ remains weak and comparable to the luminescence of pristine InP/ZnS QDs powder (Figure 4a). In particular, the emission is much weaker with respect to the same QDs dispersed in $\mathrm{CHCl}_{3}(\mathrm{Figure}$ 2d), due to an enhancement of the reabsorption processes caused by multiple light scattering. The red-shift from $624 \mathrm{~nm}$ to $684 \mathrm{~nm}$ of the QDs emission is due to a dipole-dipole energy transfer induced by the aggregation state of the QDs in their powder state. ${ }^{[36]}$ Same conclusions are made on the QD-TA-LDH compound; a red-shifted $\left(\lambda_{\mathrm{em}}=673 \mathrm{~nm}\right)$ and broader spectral signature $(\mathrm{FWHM}=82 \mathrm{~nm})$ than in the case of $\mathrm{InP} / \mathrm{ZnS}$ QDs in $\mathrm{CHCl}_{3}$ albeit with a higher intensity than the DS and $\mathrm{CO}_{3}$ compounds (Figure S7c). In contrast, QD-Bola-LDH hybrid powders show bright emission with a maximum $(636 \mathrm{~nm})$ close to the emission of InP/ZnS QDs suspended in chloroform (Figure 4b). The FWHM of the InP/ZnS QD-bola-LDH emission (184 meV) also compares well with that of well-dispersed QDs (187 meV), which confirms the efficient solid-state dilution effect provided by the bola-LDH host and the correlated narrow size distribution of the QDs (Figure S9). In a concomitant manner, the solid-state internal photoluminescent quantum yield (ssPLQY) of the QD-bola-LDH hybrid is the highest over the excitation range $300-600 \mathrm{~nm}$ (Figure 4c): the ssPLQY, amounts to $18 \%$ for 3 wt. \% of InP/ZnS QDs loaded in the bola-LDH, compared to $6.8 \%$ for pristine InP/ZnS QDs powders. The ssPLQY of the other two QD-spacer-LDH hybrid powders remains very low whatever the excitation wavelength. This clearly highlights the crucial role of guest-to-host size matching. Figure 4d accounts for the QD-loading dependence of the ssPLQY in the QD-bola-LDH system upon excitation using two wavelengths that correspond to common commercial excitation sources (see Figure S10 for ssPLQY variation over the full excitation range i.e. $300-600 \mathrm{~nm}$ ). A maximum of $18 \%$ is obtained for a loading rate of $3 \mathrm{wt} . \%$, a value that provides the most suitable dilution of the QDs within the architecture of the hybrid powder.

Moreover, as it could be seen on the absorbance spectra (Figure S11) Bola-LDH compound does not absorb light in the range of relevance (i.e. > $365 \mathrm{~nm}$ ). Since most of incident light is absorbed by the incorporated QDs and that the Bola-LDH structure does not show any fluorescence signal, no 
relevant optical synergistic effect between QDs and Bola-LDH is evidenced. The role played by the layered host is limited to the solid state dilution effect with no energetic transfer from the host matrix to the QDs.

(a)

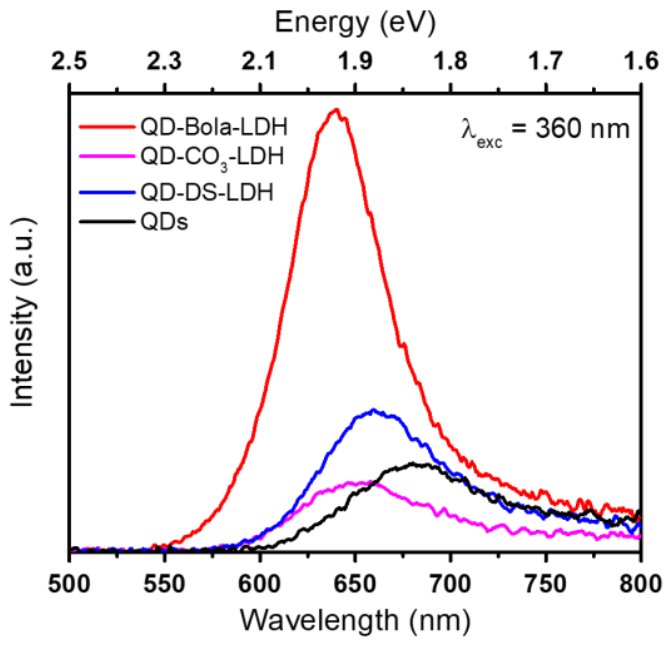

(c)

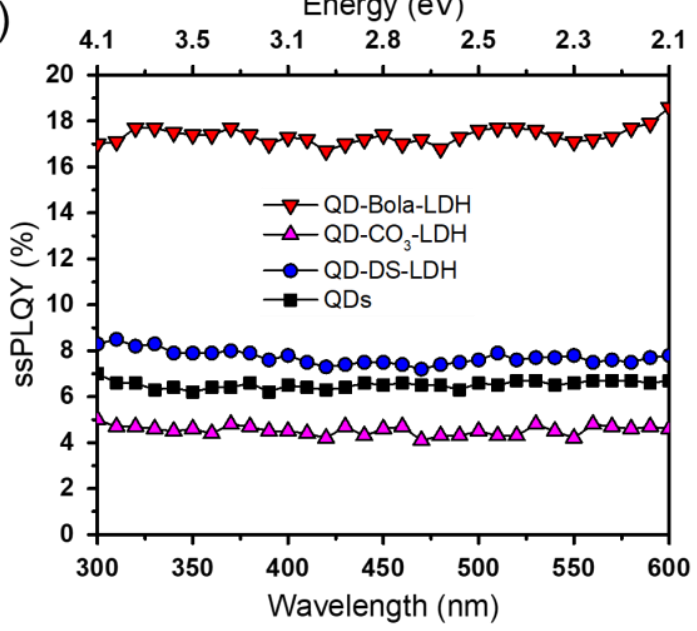

(b)

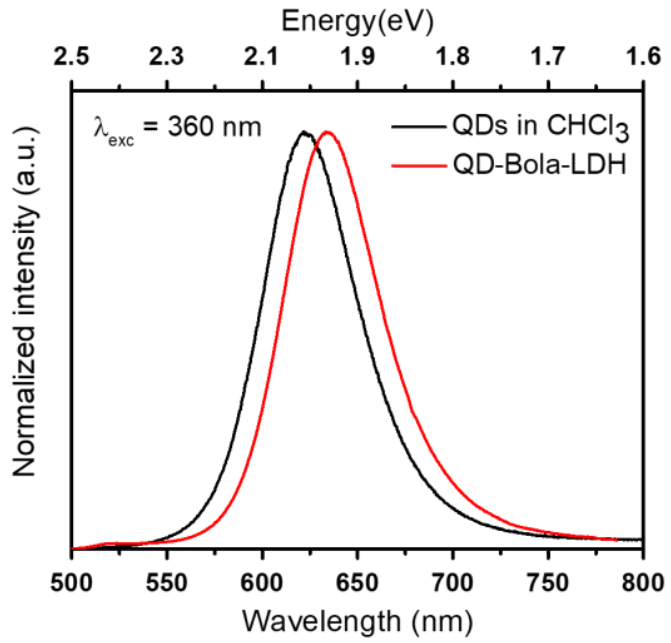

(d)

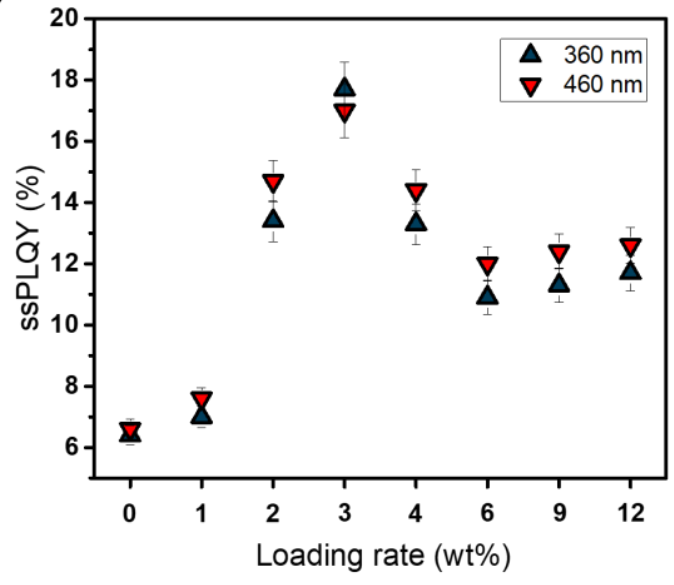

Figure 4. Emission spectra of synthesized QDs and QD-spacer-LDH powders (a), comparison between the emission spectra of QD-bola-LDH powder and of QDs in $\mathrm{CHCl}_{3}$ (b), ssPLQY of QDspacer-LDH with same QD loading rate (i.e. 3 wt. \%) (c) and comparison between several QD loading rates for QD-bola-LDH matrix at two excitation wavelengths (360 and $460 \mathrm{~nm}$ ) (d).

Fluorescence decays are investigated at room temperature to get insight of the energy transfer operating among QDs in the hybrid LDH materials. The decay profiles are displayed in the InokutiHirayama (I.-H.) model in the dipole-dipole approximation. ${ }^{[37 \mathrm{a}]}$ This model derives from the 
Förster-Dexter theory of energy transfer. ${ }^{[37 b, 37 c]}$ The corresponding data are shown Figure S12. The (I.-H.) model accounts for energy transfer in absence of migration following Equation 2,

$$
I(t)=A \exp \left(-\frac{t}{\tau}-\alpha\left(\frac{t}{\tau}\right)^{\frac{3}{n}}\right)
$$

where $I(t)$ is the luminescence intensity at time $t, \tau$ is the lifetime, $\alpha$ is the energy transfer probability and $n=6$ in the dipole-dipole approximation: ${ }^{[37 \mathrm{a}]} \tau, \alpha$ and the pre-exponential factor $\mathrm{A}$ were considered as adjustable parameters. Their values are given in Table $\mathbf{2}$ and spectra appear on Figure S12. Inspection of these data reveals that the lifetime of pristine InP/ZnS QDs, either as a powder or as a suspension in $\mathrm{CHCl}_{3}$, is the same $(\tau \sim 50 \mathrm{~ns})$, with a value consistent with the literature. ${ }^{[38 a, 38 b]}$ The $\alpha$ rate of the powder, QD-DS-LDH and QD-CO $3-\mathrm{LDH}$ is roughly two orders of magnitude larger than in the suspension, which accounts for effective energy transfer among QDs in these close-packed architectures. Conversely, the spectroscopic parameters $\tau$, $\alpha$ of QDBola-LDH hybrid compare well those of pristine QDs free in solvent and further demonstrates inefficient energy transfer in this system. This confirms the observations made previously by XRD, SAXS and absorbance spectrum concluding that the QDs are well dispersed and isolated in QDBola-LDH.

Table 2. Fitting parameters obtained for the studied compounds.

\begin{tabular}{ccc|c} 
Compounds & $\begin{array}{c}\alpha \\
\left(\mathrm{ns}^{-1}\right)\end{array}$ & $\mathrm{R}^{2}$ & $\begin{array}{c}\tau \\
(\mathrm{ns})\end{array}$ \\
\hline QD-Bola-LDH & 0.28 & 0.9993 & 51 \\
QD-DS-LDH & 7.79 & 0.9956 & 86 \\
QD-CO 3 -LDH & 3.80 & 0.9938 & 73 \\
QDs powder & 4.63 & 0.9989 & 54 \\
QDs in $\mathrm{CHCl}_{3}\left(1.5\right.$ g.L $\left.\mathrm{L}^{-1}\right)$ & 0.08 & 0.9993 & 53
\end{tabular}

The QD-spacer-LDH hybrids were encapsulated in silicone films of relevance for solid-state lighting applications to evaluate their performance (Figure S13). As illustrated by optical microscopy images, a homogeneous film is obtained with QD-bola-LDH fillers throughout the 
silicone film; QD aggregates are clearly observed and identified by EDX in the other cases (Figures S14 to S18). The homogeneity trend is QD-bola-LDH > QD-DS-LDH > QD-CO $3-\mathrm{LDH}>\mathrm{QDs}$, with QD-bola-LDH film being the most homogeneous, which was also evidenced by SEM/EDX analyses. This confirms the rather poor compatibility between InP/ZnS QDs and silicone.

Representative $120 \mu \mathrm{m}$-thick composite films are exposed to the $365 \mathrm{~nm}$ excitation of a lowpressure mercury lamp in Figure 5 and S7d. The InP/ZnS (0.27 wt. \%) QD-bola-LDH @ silicone composite clearly shows brighter and more homogeneously-distributed luminescence, as the direct consequence of the above-described microstructure. The CIE color coordinates (Figure S19) associated with the luminescence of each film further confirm the more saturated red color exhibited by InP/ZnS (0.27 wt. \%) QD-bola-LDH @ silicone film.
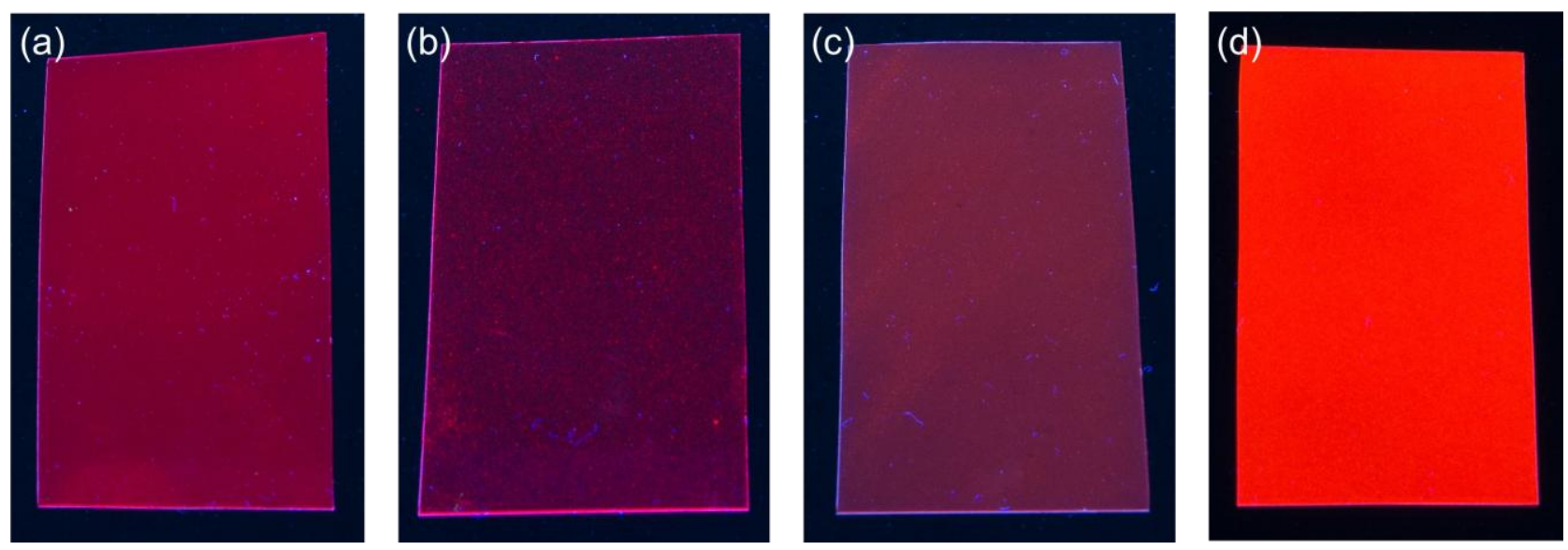

Figure 5. Image of QDs (a), QD-CO $-\mathrm{CDH}(\mathrm{b}), \mathrm{QD}-\mathrm{DS}-\mathrm{LDH}$ (c) and QD-bola-LDH (d) composite films (loading rate of QDs $=0.27 \mathrm{wt} \%)$ under UV light excitation $\left(\lambda_{\mathrm{exc}}=365 \mathrm{~nm}\right)$.

The photostability behavior of InP/ZnS (0.27 wt. \%) QD-bola-LDH @ silicone and InP/ZnS (0.27 wt. \%) QDs @ silicone films are compared in Figure 6 upon continuous 375 nm irradiation provided by a UVA LED at room temperature. The incident luminous power experienced by the samples amounted $61.2 \mathrm{~W} / \mathrm{m}^{2}$. This is a key experiment in the perspective of a further technological application. Note that the other two composite films were not tested due to a lack of photoluminescence in these experimental conditions. 
Luminescence intensity, evaluated as the integrated area below the emission spectrum, is about 6 times higher for the InP/ZnS (0.27 wt. \%) QD-bola-LDH @ silicone composite. A $\approx 20 \%$ rise in the initial emission intensity is observed in this sample. Similar behavior was observed in several other structures based on $\mathrm{PbS}{ }^{[39]}$ and $\mathrm{CdSe}{ }^{[40]} \mathrm{QDs}$, and is explained by the adsorption of water molecules onto the QD surface or by the rearrangement of the surface ligands. The latter seems unlikely in the present case, however, because of the presence of silicone immobilizing the QDs in the LDH interlayer space. A focus on the time-dependence of emission spectra in the early stages of illumination (i.e. $<4 \mathrm{~h}$, see Figure 6) reveals a blue shift of $\approx 8 \mathrm{~nm}$ of the emission band (Figure S20), which is associated with a shrinkage of the InP core. This phenomenon is ascribed to the diffusion of water and oxygen molecules due to the mismatched interface $(8 \%$ in terms of cell parameter) between the InP core and the $\mathrm{ZnS}$ shell, followed by local photo-oxidation processes. ${ }^{\text {[40] }}$ Despite the faster rate of photo-induced quenching of the luminescence in the $\mathrm{InP} / \mathrm{ZnS}(0.27$ wt. \%) QD-bola-LDH@ @ilicone composite, the emitted intensity still remains about 3 times that of InP/ZnS (0.27 wt. \%) QD @ silicone composite. This demonstrates the efficient shielding character of the bola-LDH architecture vs. the deleterious action of external irradiation on the InP/ZnS QDs.

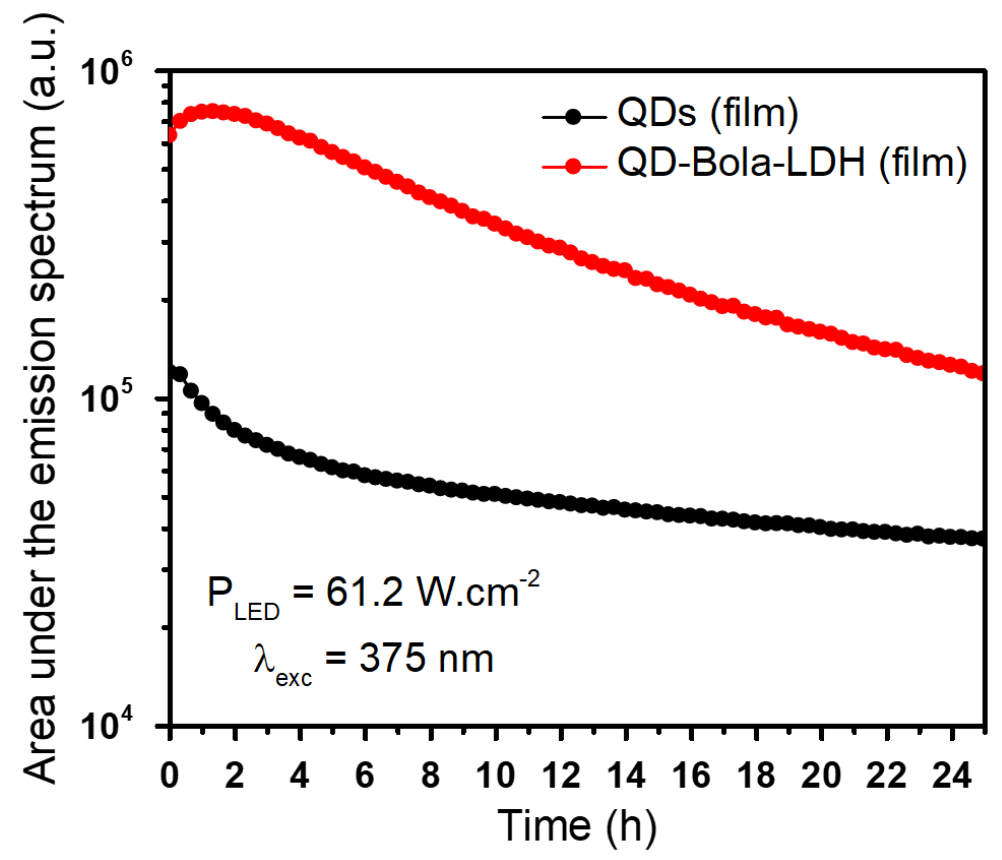


Figure 6. Variation in the integrated areas of the emission spectra as a function of irradiation time for QD-bola-LDH (red plot) and QDs (black plot) composite films (loading rate 0.27 wt. \%).

Moving to W-LED solid-state lighting applications, composite films of silicone-containing commercial YAG-Ce ${ }^{3+}$ and InP/ZnS (1 wt. \%) QD-bola-LDH phosphors were combined with a $465 \mathrm{~nm}$ commercial LED in remote mode with the objective of lowering the colour temperature (CT) and increasing the colour rendering index (CRI) of the device with respect to the cold-white emitting blue LED + YAG-Ce ${ }^{3+}$ system (Figure 7). The photometric parameters of the different configurations shown in Figure 7 were compiled. For configuration 1, the emission is centered on the greenish-yellow spectral range and the overall produced color corresponds to cool white with parameters $\mathrm{CT}=6144 \mathrm{~K}$ and CRI 76.7. When the red-emitting QD-bola-LDH composite film is added, as shown in configuration 2 , the photometric parameters change to $\mathrm{CT}=5410 \mathrm{~K}$ and $\mathrm{CRI}=$ 82.2, which gives warmer white light. Configuration 3 yields $\mathrm{CT}=4648 \mathrm{~K}$ and $\mathrm{CRI}=84.9$, which is significantly warmer. The difference is due to the fact that the emission produced by the YAG: $\mathrm{Ce}^{3+}$ film is reabsorbed by the QD-bola-LDH composite film in this configuration, which thus reinforces the contribution of the red component to the global spectrum. Photometric parameters CT $=3576 \mathrm{~K}$ and $\mathrm{CRI}=93.8$ are finally reached when adding a second $120 \mu \mathrm{m}$-thick QD-bola-LDH film as in configuration 4. This fulfils expectations of a further breakthrough in the W-LED field. 
(a)

(b)

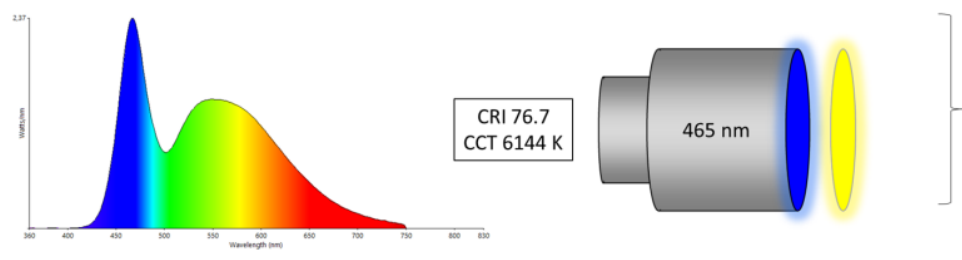

Configuration 2

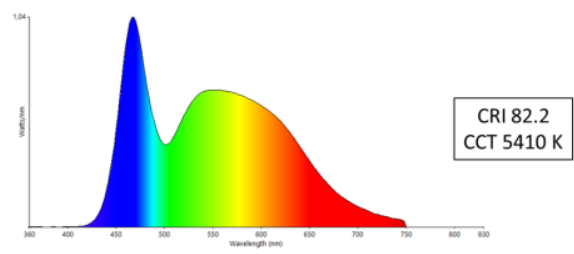

(c) Configuration 3

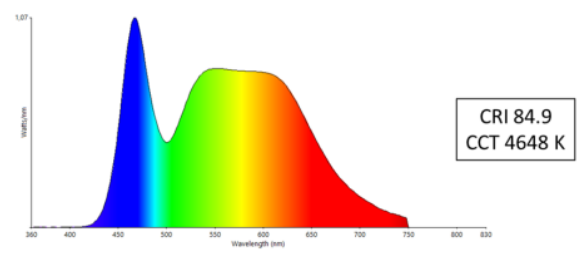

(d) Configuration 4

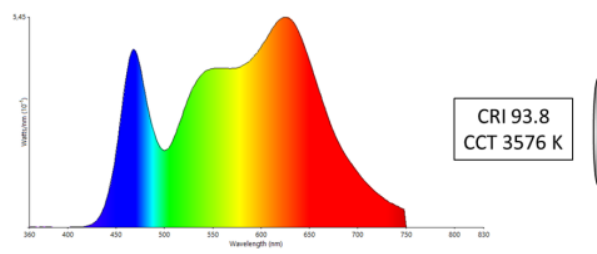

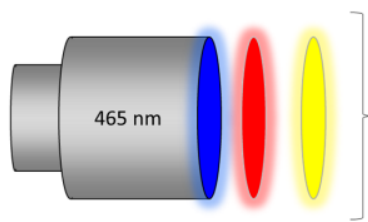

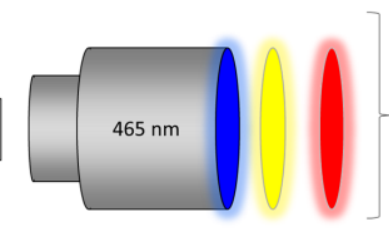

Blue LED

QD-bola-LDH silicone film (1 wt \%)

YAG silicone film (30 wt \%)

\section{Blue LED}

YAG silicone film $(30 \mathrm{wt} \%)$ QD-bola-LDH silicone film (1 wt\%) QD-bola-LDH silicone film (1 wt\%)

Figure 7. Emission spectra, photometric parameters and designed white LED prototypes implemented for configuration 1 (a), 2 (b), 3 (c) and 4 (d).

To recapitulate, the SMIS method using a bi-tethered spacer such as bola-type molecule is demonstrated to be original and straightforward to accommodate controllable amounts of core-shell InP/ZnS QDs within the interlamellar space of a LDH host structure. Focusing on the size-matching of the bi-tethered spacer and its QD guest nanoentities is preferred to ensure the incorporation of QDs into the interlamellar space of the LDH. The resulting highly promising red-emitting InP/ZnS QD-bola-LDH hybrid powder phosphor shows an emission peak at the same wavelength and as narrow as the QDs dispersed in a solution. The controlled entrapping of the InP/ZnS QDs within the hybrid bola-LDH host results in optimized solid-state dilution, significant enhancement of the ssPLQY without QD-QD energy transfer and improved photo-stability. Furthermore, the SMIS 
method paves the way towards the production of synergistic QD-LDH systems which are easy to disperse in polymers and show efficient and sustainable optical properties; this is barely feasible using LB or LbL strategies. As a proof of concept, a LED-based structure consisting of a blue LED chip combined in remote mode with silicone films loaded with $\mathrm{InP} / \mathrm{ZnS}$-bola-LDH hybrids and commercial YAG: $\mathrm{Ce}^{3+}$ phosphors was qualified with a color temperature of $3576 \mathrm{~K}$ and a color rendering index of 93.8 , thus opening very promising perspectives in terms of lighting applications. 


\section{Experimental Section}

All synthetic details (QDs, LDH matrices and QD-LDH composites), along with all the film formation procedures and the devices used for each characterization are described in the Supporting Information.

\section{Supporting Information}

\section{Acknowledgements}

R.V. and Q.Z. contributed equally to the present work. The authors would like to acknowledge the Centre Imagerie Cellulaire Sante (CICS) (UCA PARTNER - Clermont-Ferrand, FRANCE) and 2MAtech, for their technical support and expertise on TEM and FEG/SEM analyses. Thomas Lohmeier, former working student at BASF Coatings Division, for the supply of bola-amphiphiles. Q.Z. gratefully acknowledges the National Natural Science Foundation of China (No. U1707603) and the financial support from China Scholarship Council (No. 201906880038). 


\section{References}

[1] P. Gin, M. Bruchez, A. P. Alivisatos, M. Moronne, S. Weiss, Science (80-. ). 1998, 281, 2013.

[2] P. Geiregat, D. Van Thourhout, Z. Hens, NPG Asia Mater. 2019, 11, 41.

[3] E. Jang, Y. Kim, Y. H. Won, H. Jang, S. M. Choi, ACS Energy Lett. 2020, 5, 1316.

[4] F. Cao, S. Wang, F. Wang, Q. Wu, D. Zhao, X. Yang, Chem. Mater. 2018, 30, 8002.

[5] O. I. Mićić, J. R. Sprague, C. J. Curtis, K. M. Jones, J. L. Machol, A. J. Nozik, H. Giessen, B. Fluegel, G. Mohs, N. Peyghambarian, J. Phys. Chem. 1995, 99, 7754.

[6] W. S. Song, H. S. Lee, J. C. Lee, D. S. Jang, Y. Choi, M. Choi, H. Yang, J. Nanoparticle Res. 2013, $15,1750$.

[7] K. R. Reid, J. R. McBride, N. J. Freymeyer, L. B. Thal, S. J. Rosenthal, Nano Lett. 2018, 18, 709.

[8] D. Hahm, J. H. Chang, B. G. Jeong, P. Park, J. Kim, S. Lee, J. Choi, W. D. Kim, S. Rhee, J. Lim, D. C. Lee, C. Lee, K. Char, W. K. Bae, Chem. Mater. 2019, 31, 3476.

[9] Y. H. Won, O. Cho, T. Kim, D. Y. Chung, T. Kim, H. Chung, H. Jang, J. Lee, D. Kim, E. Jang, Nature 2019, 575, 634.

[10] S. Kim, T. Kim, M. Kang, S. K. Kwak, T. W. Yoo, L. S. Park, I. Yang, S. Hwang, J. E. Lee, S. K. Kim, S. W. Kim, J. Am. Chem. Soc. 2012, 134, 3804.

[11] T. Wu, X. Liu, Y. Liu, M. Cheng, Z. Liu, G. Zeng, B. Shao, Q. Liang, W. Zhang, Q. He, Coord. Chem. Rev. 2020, 403, 1.

[12] J. Aguilera-Sigalat, D. Bradshaw, Coord. Chem. Rev. 2016, 307, 267.

[13] M. C. So, G. P. Wiederrecht, J. E. Mondloch, J. T. Hupp, O. K. Farha, Chem. Commun. 2015, 51,3501 .

[14] S. Cho, J. Kwag, S. Jeong, Y. Baek, S. Kim, Chem. Mater. 2013, 25, 1071.

[15] R. Liang, D. Yan, R. Tian, X. Yu, W. Shi, C. Li, M. Wei, D. G. Evans, X. Duan, Chem. Mater. 2014, 26, 2595.

[16] J. S. Bendall, M. Paderi, F. Ghigliotti, N. L. Pira, V. Lambertini, V. Lesnyak, N. Gaponik, G. 
Visimberga, A. Eychmüller, C. M. S. Torres, M. E. Welland, C. Gieck, L. Marchese, Adv. Funct. Mater. 2010, 20, 3298.

[17] R. Liang, S. Xu, D. Yan, W. Shi, R. Tian, H. Yan, M. Wei, D. G. Evans, X. Duan, Adv. Funct. Mater. 2012, 22, 4940.

[18] S. Cho, S. C. Hong, S. Kim, J. Mater. Chem. C 2014, 2, 450.

[19] J. Ren, T. Li, X. Zhou, X. Dong, A. V. Shorokhov, M. B. Semenov, V. D. Krevchik, Y. Wang, Chem. Eng. J. 2019, 358, 30.

[20] R. Ma, Z. Tian, Y. Hu, Y. Huang, J. Lu, Langmuir 2018, 34, 11354.

[21] B. R. Venugopal, N. Ravishankar, C. R. Perrey, C. Shivakumara, M. Rajamathi, J. Phys. Chem. B 2006, 110, 772 .

[22] a) J. Cortese, C. Soulié-Ziakovic, M. Cloitre, S. Tencé-Girault, L. Leibler, J. Am. Chem. Soc. 2011, 133, 19672. b) T. Kato, N. Mizoshita, K. Kishimoto, Angewandte Chemie International Edition 2005, 45, 38. c) M. Sofos, D. A. Stone, D. K. Goswami, J. S. Okasinski, H. Jin, M. J. Bedzyk, S. I. Stupp, J. Phys. Chem. C 2008, 112, 2881.

[23] a) A. Langry, J. Cellier, H. Hintze-Bruening, F. Leroux, Dalt. Trans. 2018, 47, 3005. b) T. Lohmeier, M. Bredol, E. Schreiner, H. Hintze-Bruening, Soft Matter 2014, 10, 6237.

[24] M. A. Drezdzon, Inorg. Chem. 1988, 27, 4628.

[25] S. Tamang, G. Beaune, I. Texier, P. Reiss, ACS Nano 2011, 5, 9392.

[26] M. D. Tessier, D. Dupont, K. De Nolf, J. De Roo, Z. Hens, Chem. Mater. 2015, 27, 4893.

[27] Y. Kim, J. H. Chang, H. Choi, Y. H. Kim, W. K. Bae, S. Jeong, Chem. Sci. 2020, 11, 913.

[28] HWHM: Half-Width at Half-Maximum, used instead of FHWM since one can only experimentally deduce half of the absorption peak.

[29] N. E. Williams, D. J. Gosztola, L. Wang, C. Sun, E. M. Janke, C. She, D. V. Talapin, R. D. Schaller, G. S. Engel, B. Lee, D. Zherebetskyy, M. H. Hudson, J. Am. Chem. Soc. 2018, 140, 15791.

[30] G. Xu, S. Zeng, B. Zhang, M. T. Swihart, K. T. Yong, P. N. Prasad, Chem. Rev. 2016, 116, 
12234.

[31] G. Laufersky, S. Bradley, E. Frécaut, L. Matthias, T. Nann, Nanoscale 2018, 7, 332.

[32] a) C. Taviot-Guého, V. Prevost, C. Forano, G. Renaudin, C. Mousty, F. Leroux, Adv. Func. Mat. 2017, 1703868. b) J.-Y. Liu, G.-C. Lv, W.-L. Gu, Z.-H. Li, A.-W. Tang, L.-F. Mei, J. Mater. Chem. C 2017, 5, 5024. c) Y.-Q. Tang, R.-R. Wang, Y. Yang, D.-P. Yan, X. Xiang, ACS Appl. Mater. Interfaces 2016, 8, 19446. d) S. cho, S. Jung, S. Jeong, J. Bang, J. Park, Y. Park, S. Kim, Langmuir 2013, 29, 441-447.

[33] M. Meyn, K. Beneke, G. Lagaly, Inorg. Chem. 1990, 29, 5201.

[34] R. Anderson, R. Buscall, R. Elridge, P. Mulvaney, P. Scales, RSC Adv. 2014, 4, 46876.

[35] J. Plank, K. Poellmann, N. Zouaoui, P. R. Andres, C. Schaefer, Cement Concrete Res. 2008, 38,1210

[36] U. T. D. Thuy, P. T. Thuy, N. Q. Liem, L. Li, P. Reiss, Appl. Phys. Lett. 2010, 96, 1.

[37] a) M. Inokuti, F. Hirayama, J. Chem. Phys. 1965, 43, 1978-1989. b) D. L. Dexter, J. Chem. Phys. 1953, 21, 836. c) Th. Förster, Z. Naturforsch. A4. 1949, 5, 321-327.

[38] a) M. Lim, W. Lee, G. Bang, W. J. Lee, Y. Park, Y. Kwon, S. Kim, J. Bang, Nanoscale 2019, 11, 10463. b) C. K. De, T. Routh, D. Roy, S. Mandal, P. K. Mandal, J. Phys. Chem. C 2018, 122, 964-973.

[39] M. S. Zaini, J. Ying, C. Liew, S. Ainliah, A. Ahmad, A. R. Mohmad, M. A. Kamarudin, Appl. Sci. 2020, 10, 6282.

[40] C. Carrillo-Carrión, S. Cárdenas, B. M. Simonet, M. Valcárcel, Chem. Commun. 2009, 5214. 